\title{
Regulation of steroid hormones in the placenta and serum of women with preeclampsia
}

\author{
YE YOUNG SHIN ${ }^{1}$, JEA SIC JEONG ${ }^{1}$, MEE-NA PARK ${ }^{1}$, JAE-EON LEE $^{1}$, SUNG-MIN AN $^{1}$, \\ WAN-SEOB CHO ${ }^{2}$, SEUNG CHUL KIM ${ }^{3}$, BEUM-SOO AN ${ }^{1}$ and KYU-SUP LEE ${ }^{3}$ \\ ${ }^{1}$ Department of Biomaterials Science, College of Natural Resources and Life Science/Life and \\ Industry Convergence Research Institute, Pusan National University, Miryang, Gyeongsangnam 627-706; \\ ${ }^{2}$ Department of Medicinal Biotechnology, School of Natural Resources and Life Science, Dong-A University, \\ Busan, Gyeongsangnam 49315; ${ }^{3}$ Department of Obstetrics and Gynecology, Biomedical Research Institute, \\ Pusan National University School of Medicine, Busan, Gyeongsangnam 49241, Republic of Korea
}

Received April 20, 2017; Accepted October 2, 2017

DOI: $10.3892 / \mathrm{mmr} .2017 .8165$

\begin{abstract}
Preeclampsia (PE) is a pregnancy-specific hypertensive syndrome that results in substantial maternal and fetal morbidity and mortality. The exact cause of PE has not been completely elucidate, although abnormal formation of the placenta has been considered. The placenta connects the developing fetus to the uterine wall, producing a large quantity of steroid hormones to maintain pregnancy. Although steroid hormones, particularly progesterone (P4) and estrogen (E2), in the serum of women with PE have been studied, steroidogenesis in the placenta has not well been established. The present study compared the concentrations of steroid hormones, including pregnenolone (PG), P4, dehydroepiandrosterone (DHEA), testosterone (T) and E2, in the serum and placenta of women with PE. PG, P4, DHEA and E2 concentrations tended to be decreased in PE serum and placentas, and the results were statistically significant for $\mathrm{P} 4$ and $\mathrm{E} 2$ in the serum. Quantification of genes associated with steroidogenesis in the placenta was performed, and the expression of the P4- and E2-synthesizing enzymes testosterone $17-\beta$-dehydrogenase 3 and $3 \beta$-hydroxysteroid dehydrogenase/ $\delta 54$-isomerase type
\end{abstract}

Correspondence to: Professor Kyu-Sup Lee, Department of Obstetrics and Gynecology, Biomedical Research Institute, Pusan National University School of Medicine, 179 Gudeok-ro, Seo-gu, Busan, Gyeongsangnam 49241, Republic of Korea

E-mail:kuslee@pusan.ac.kr

Professor Beum-Soo An, Department of Biomaterials Science, College of Natural Resources and Life Science/Life and Industry Convergence Research Institute, Pusan National University, 50 Cheonghak-ri, Samrangjin-eup, Miryang, Gyeongsangnam 627-706, Republic of Korea

E-mail: anbs@pusan.ac.kr

Key words: placenta, serum, preeclampsia, steroid hormones, steroidogenesis
1 was reduced. Notably, aromatase, an enzyme required for the production of E2, was upregulated in the PE placenta, suggesting that steroidogenic enzymes may be dynamically regulated and may affect the symptoms of PE. In conclusion, the results of the present study suggested that the levels of steroid hormones, including P4 and E2, in the serum and placenta of women with PE are downregulated, which may be mediated by the regulation of steroidogenic enzyme expression in the PE placenta.

\section{Introduction}

Preeclampsia $(\mathrm{PE})$ is a pregnancy-specific hypertensive syndrome affecting $2-7 \%$ of pregnant women globally, developing subsequent to 20 weeks of gestational age (1). The clinical manifestations of PE include hypertension, placental hypoxia, proteinuria, endothelial dysfunction, end-organ ischemia and increased vascular permeability (2). These conditions are the leading causes of maternal and fetal morbidity and mortality (3).

Although there have been previous studies into the mechanism of $\mathrm{PE}$, its exact pathogenic mechanism remains unknown (4). It has been widely accepted that dysfunction of the placenta, which is the organ facilitating the exchange of nutrients and waste between the mother and fetus, may result in PE (5). The placenta begins to develop upon invasion of the blastocyst into the endometrium. The outer layer of the blastocyst becomes the trophoblast, which serves an essential role in the formation of the placenta. During normal pregnancy, appropriate trophoblast invasion produces spiral arteries to facilitate the exchange of gases between mother and fetus (6). However, PE begins with incomplete trophoblast invasion at the early stages of pregnancy, which disrupts correct placental formation. A reduction of trophoblastic invasion at the decidual and myometrial levels, and a failure of trophoblast cells to replace the spiral artery, have been observed in the PE placenta (7). High blood pressure with low blood flow are generated in PE, resulting in reduced uteroplacental blood flow. Incomplete placentation additionally produces increased placental oxidative stress, contributing to the development 
of systemic endothelial dysfunction in later phases of the disease (8).

During pregnancy, the placenta is the primary endocrine organ for maintaining pregnancy and fetal growth. Hormones released by the placenta regulate the growth and differentiation of the placental trophoblast, growth and maturation of the placental vascular tree, and uterine endovascular invasion by the extravillous cytotrophoblast (9). To successfully establish pregnancy, a number of steroid hormones are synthesized and secreted through steroidogenesis in the placenta. Progesterone (P4) and estrogen (E2) are the principal steroid hormones produced by the placenta in primate pregnancy (10). The serum levels of P4 and E2 increase throughout pregnancy (11).

Steroidogenesis is the biological process through which cholesterol is converted into multiple steroid hormones (12). The pathway of steroidogenesis is mediated by steroidogenic enzymes (13). The conversion of cholesterol into pregnenolone (PG) by cholesterol side-chain cleavage enzyme (CYP11A1) represents the initiation of steroidogenesis (14). Following its production, $\mathrm{PG}$ is converted into $\mathrm{P} 4$ or dehydroepiandrosterone (DHEA) by $3 \beta$-hydroxysteroid dehydrogenase/ $\delta 54$-isomerase type 1 (HSD3B1) or steroid 17- $\alpha$-hydroxylase/17,20 lyase (CYP17A1), respectively. Androgens, including testosterone (T), may be synthesized from DHEA and P4, mediated by testosterone $17-\beta$-dehydrogenase 3 (HSD17B3) and HSD3B1 (15). Aromatase (CYP19A1) and HSD17B3 catalyze the final steps of E2 biosynthesis from androgens. These steps require a number of factors and complex processes comprising networks of intracellular signaling pathways (16).

In previous studies, the levels of steroid hormones in patients with PE have been compared with those in normal pregnant women to reveal the mechanism of PE $(17,18)$. However, only the levels in serum have been focused on, while the placental steroid concentrations and comparisons with those from serum have not been addressed. Therefore, the present study investigated the expression of steroidogenic enzymes and analyzed the concentration of steroid hormones in the placenta and serum. The association between PE and steroid hormones from the placenta and serum may provide insights into the pathophysiological characteristics. In addition, the results of the present study may suggest potential biomarkers to predict $\mathrm{PE}$ and possible therapeutic methods to treat women with PE.

\section{Materials and methods}

Tissue and blood collection. Human placental tissues and blood samples were collected and immediately stored at $-80^{\circ} \mathrm{C}$, which were divided into normal women $(\mathrm{n}=21)$ and patients with PE $(n=20)$. The samples were obtained between 29 and 40 weeks of gestation from Jan to Dec of 2015 and provided by the Biobank of Pusan National University Hospital (Busan, Korea), a member of the Korea Biobank Network. The present study was approved by the Institutional Review Board of the Pusan National University Hospital Clinical Trials Center (no. H-1302-005-015), and all participants gave written informed consent. Patients with PE were defined as having systolic and diastolic blood pressures $>140$ and $90 \mathrm{~mm} \mathrm{Hg}$, respectively, measured at least
$6 \mathrm{~h}$ apart, in addition to proteinuria $>300 \mathrm{mg} / 24 \mathrm{~h}$ or $>1+$ as determined by the dipstick method. Blood was collected in plastic tubes under aseptic conditions with EDTA as an anti-coagulant and centrifuged at $18,472 \mathrm{x}$ g for $10 \mathrm{~min}$ at $4^{\circ} \mathrm{C}$ in order to separate the serum. Clinical details of the patients are presented in Table I.

Reverse transcription-quantitative polymerase chain reaction (RT-qPCR) analysis. Total RNA from placenta tissue was extracted using TRIzol reagent (Invitrogen; Thermo Fisher Scientific, Inc., Waltham, MA, USA), according to the manufacturer's protocol. The concentration of total RNA was measured using a spectrophotometer. First-strand cDNA was prepared from total RNA (3 $\mu \mathrm{g})$ via reverse transcription at $37^{\circ} \mathrm{C}$ using Moloney murine leukemia virus reverse transcriptase (Invitrogen; Thermo Fisher Scientific, Inc.) and random primers (9-mers; Takara Bio Inc., Otsu, Japan), according to the manufacturer's instructions and stored at $-20^{\circ} \mathrm{C}$ until use. qPCR was performed with the cDNA template $(2 \mu \mathrm{l})$ and $2 \mathrm{X}$ Power SYBR Green ( $6 \mu$ l; Toyobo Life Science, Osaka, Japan) containing specific primers. Primer sequences for cytochrome $\mathrm{c} 1$ heme protein mitochondrial (CYC1), $\beta$-actin, CYP11A1, CYP17A1, HSD17B3, HSD3B1, and CYP19A1 are presented in Table II. qPCR was performed for 40 cycles using the following parameters: Denaturation at $95^{\circ} \mathrm{C}$ for $15 \mathrm{sec}$, followed by annealing and extension at $70^{\circ} \mathrm{C}$ for $60 \mathrm{sec}$. Fluorescence intensity was measured at the end of the extension phase of each cycle. The threshold value for the fluorescence intensities of all samples was set manually. The reaction cycle at which the PCR products exceeded this fluorescence intensity threshold during the exponential phase of PCR amplification was considered to be the threshold cycle (Cq). Expression of the target gene was quantified relative to that of $\mathrm{CYC1}$ and $\beta$-actin, which are housekeeping genes, based on comparison of the Cqs at a constant fluorescence intensity as previously described (19).

Western blot analysis. Protein samples of the placenta tissues were extracted with Pro-prep solution (Intron Biotechnology, Inc., Seongnam, Korea), according to the manufacturer's protocol. The concentration of the protein was determined via a bicinchoninic acid assay; a total of $20 \mu \mathrm{g}$ protein, was loaded and separated by SDS-PAGE on an 8-10\% gel and transferred onto nitrocellulose membranes (Daeillab Lab Service Co., Ltd., Seoul, Korea). Membranes were blocked for $2 \mathrm{~h}$ with 5\% skimmed milk (Difco ${ }^{\mathrm{TM}}$; BD Biosciences, Franklin Lakes, NJ, USA) in PBS with $0.05 \%$ Tween-20 (PBS-T) at room temperature. Following blocking, membranes were incubated with anti-CYP11A1 (1:100; cat. no. sc-18040, Santa Cruz Biotechnology, Inc., Dallas, TX, USA), CYP17A1 (1:500; cat. no. sc-46084, Santa Cruz Biotechnology, Inc.), HSD17B3 (1:2,000; cat. no. ab55268, Abcam, Cambridge, UK), HSD3B1 (1:2,000; sc-30820, Santa Cruz Biotechnology, Inc.), and CYP19A1 (1:500; cat no. sc-14244, Santa Cruz Biotechnology, Inc.) antibodies overnight at $4^{\circ} \mathrm{C}$, followed by horseradish peroxidase-conjugated secondary antibodies (all 1:2,000; cat. nos. sc-2313, sc-2005, sc-2020, Santa Cruz Biotechnology, Inc.) in 5\% skimmed milk with PBS-T for $1 \mathrm{~h}$ at room temperature. Luminol reagent (Bio-Rad Laboratories, Inc., Hercules, CA, USA) was used to visualize antibody binding. Membranes 
Table I. Clinical characteristics of pregnancy women.

\begin{tabular}{lccc}
\hline Characteristic & Normal $(\mathrm{n}=21)$ & Preeclampsia $(\mathrm{n}=20)$ & P-value \\
\hline Age, years & $31.6 \pm 3.58$ & $33.9 \pm 3.05$ & N.S \\
Gestational age, weeks & $35.1 \pm 1.8$ & $35.6 \pm 2.6$ & N.S \\
Weight, $\mathrm{kg}$ & $66.1 \pm 8.4$ & $74.9 \pm 14.0$ & $<0.05$ \\
BMI, $\mathrm{kg} / \mathrm{m}^{2}$ & $25.8 \pm 3.0$ & $28.6 \pm 5.8$ & $\mathrm{~N} . \mathrm{S}$ \\
Systolic BP, $\mathrm{mmHg}$ & $107.1 \pm 7.8$ & $147.9 \pm 12.7$ & $<0.05$ \\
Diastolic BP, $\mathrm{mmHg}$ & $67.1 \pm 7.2$ & $96.8 \pm 10.0$ & $<0.05$ \\
Parity & $0.8 \pm 0.7$ & $0.6 \pm 0.7$ & N.S \\
Gravidity & $2.6 \pm 1.4$ & $2.3 \pm 1.3$ & N.S \\
Maternal BMI, $\mathrm{kg} / \mathrm{m}^{2}$ & $15.8 \pm 3.0$ & $28.6 \pm 5.8$ & N.S \\
Infant birth weight, g & $2485.7 \pm 439.3$ & $2130.0 \pm 686.2$ & $<0.05$
\end{tabular}

BMI, body mass index; BP, blood pressure; N.S, not significant. Data are presented as the mean \pm standard deviation.

Table II. Primer sequences for the reverse transcription-quantitative polymerase chain reaction.

\begin{tabular}{lll}
\hline Gene name & Orientation & \multicolumn{1}{c}{ Sequence, 5'- 3' } \\
\hline$\beta$-actin & Forward & GGagment, bp \\
CYC1 & Reverse & AGCACTGTGTTGGCGTACAG \\
& Forward & CCAGCTACCATGTCCCAGAT \\
CYP11A1 & Reverse & TATGCCAGCTTCCGACTCTT \\
& Forward & GCAACGTGGAGTCGGTTTAT \\
CYP17A1 & Reverse & ATTGCAGCATCTTGCTTGTG \\
& Forward & CTGATGCAAGCCAAGATGAA \\
HSD17B3 & Reverse & GCTGAAACCCACATTCTGGT \\
HSD3B1 & Forward & ATCCAGAGCCTCATCCATTG \\
& Reverse & AACGCCTTGGAAGCTGAGTA \\
CYP19A1 & Forward & AGAGGCCTGTGTCCAAGCTA \\
& Reverse & TTTTGCTGTGTGGGTATGGA \\
\hline
\end{tabular}

CYP11A1, cholesterol side-chain cleavage enzyme; CYP17A1, steroid 17- $\alpha$-hydroxylase/17,20 lyase; HSD17B3, testosterone 17- $\beta$-dehydrogenase 3; HSD3B1, 3 $\beta$-hydroxysteroid dehydrogenase/ 85 4-isomerase type 1; CYP19A1, aromatase; CYC1, cytochrome c1 heme protein mitochondrial.

were subsequently probed with an antibody against $\beta$-actin (Cell Signaling Technology Inc., Danvers, MA, USA; diluted 1:3,000; cat. no. 967) as an internal control. Blots were scanned using Gel Doc 1000, version 1.5 (Bio-Rad Laboratories, Inc.), and band intensities were normalized to $\beta$-actin levels.

ELISA analysis. Concentrations of PG (cat. no. KA1912, Abnova, Taoyuan, Taiwan)), P4 (cat. no. 582601; Cayman Chemical Company, Ann Arbor, MI, USA;), DHEA (cat. no. 20-DHEHU-E01, Enzo Life Sciences, Inc., Farmingdale, NY, USA; ), T (cat. no. 582701; Cayman Chemical Company), and E2 (cat. no. 582251; Cayman Chemical Company) were measured using competitive enzyme immunoassay kits, according to the manufacturers' protocols. Serum and tissues were added to a 96-well plate following tissue homogenization with Pro-prep solution (Intron biotechnology, Seoul, South Korea), according to the manufacturer's protocols. The plate was incubated for $1 \mathrm{~h}$ at room temperature on an orbital shaker. Following incubation at room temperature for 90 min with Ellman's reagent, optical density values were measured spectrophotometrically at $420 \mathrm{~nm}$. The final concentrations were calculated using standard curve analysis.

Statistical analysis. Results are presented as the mean \pm standard deviation. Data were analyzed using Sigma Plot 10.0 (Systat Software, Inc., San Jose, CA, USA). Repeats were performed $\geq 3$ times; differences between groups were calculated using a Student's t-test. $\mathrm{P}<0.05$ was considered to indicate a statistically significant difference. 
A

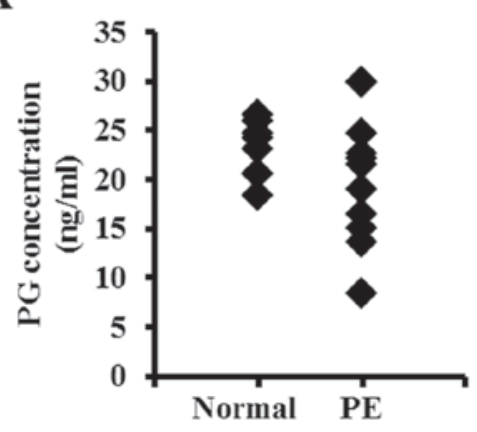

C

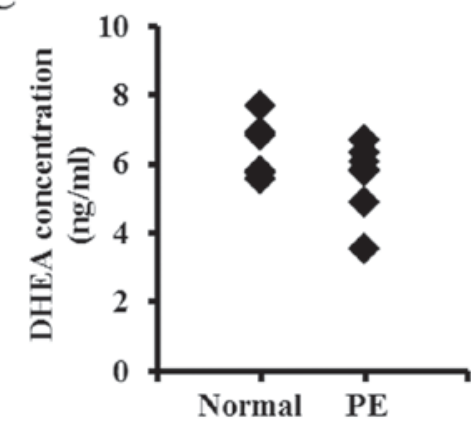

D

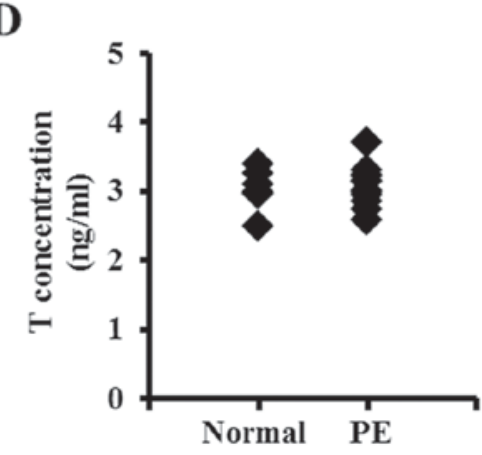

B

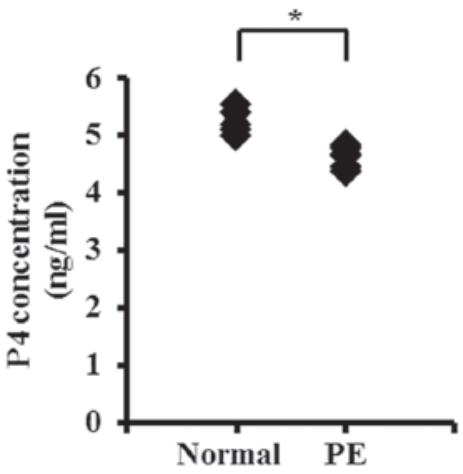

E

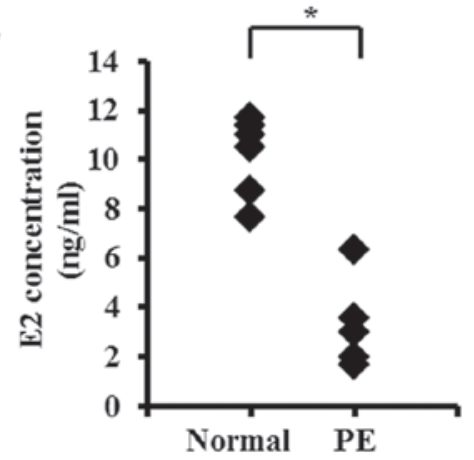

Figure 1. Serum concentration of steroid hormones in women with PE. Serum steroid hormone concentrations of (A) PG, (B) P4, (C) DHEA, (D) T and (E) E2 were analyzed by ELISA, comparing normal serum with PE serum. "P<0.05 vs. Normal. PE, preeclampsia; PG, pregnenolone; P4, progesterone; DHEA, dehydroepiandrosterone; T, testosterone; E2, estrogen.

\section{Results}

Concentration of steroid hormones in PE serum and placenta. The characteristics of the patients with PE are summarized in Table I. Patients with PE exhibited high blood pressure $(>140 / 90 \mathrm{mmHg})$, and the average blood pressure was $147.9 \pm 12.7 / 96.8 \pm 10.0 \mathrm{mmHg}$. Patients with PE exhibited an increased body weight, while infant weight was markedly decreased. Parity, gravidity and maternal body mass index were not notably different between normal patients and patients with PE, which was consistent with other studies $(20,21)$. To investigate the association between steroid hormones and PE, the concentrations of steroid hormones were evaluated to verify whether or not the PE placenta is under different endocrinal conditions compared with the normal placenta. The concentrations of PG, P4, DHEA, T and E2 in the serum and placenta were analyzed using ELISA kit (Figs. 1 and 2). Serum levels of PG, P4, DHEA and E2 tended to decline in women with PE (with significant results for $\mathrm{P} 4$ and E2), whereas the concentrations of $\mathrm{T}$ did not exhibit any significant difference in PE compared with normal serum. The concentrations of PG, DHEA and E2 in the serum were increased compared with the placenta, whereas the levels of P4 and T were similar. Hormonal levels in placental tissues exhibited similar results compared with the serum. Levels of PG, P4, DHEA and E2 were reduced in the PE placenta, although the results were not significant.

Expression of steroidogenic enzymes in the PE placenta. Since the concentrations of steroid hormones were altered in PE serum and placentas, the expression of steroidogenic enzymes in the PE placenta was subsequently investigated.
The mRNA expression of CYP11A1, CYP17A1, HSD17B3, HSD3B1 and CYP19A1, which are salient steroidogenic enzymes, were examined by RT-qPCR analysis (Fig. 3). The mRNA level of HSD3B1 was decreased in PE compared with the normal placenta, whereas that of CYP19A1 was elevated by 2 -fold in the PE placenta (Fig. 3D and E). Transcription levels of other steroidogenic enzymes were not significantly altered. The translation levels of steroidogenic enzymes were analyzed by western blotting (Fig. 4). The protein levels of HSD3B1 exhibited similar patterns compared with the mRNA by significantly decreasing (Fig. 4A). The CYP19A1 protein level increased, although the result was not statistically significant (Fig. 4D). CYP11A1 and CYP17A1 mRNA levels were unaltered in the PE group (Fig. 4A and B). Notably, the protein level of HSD17B3 was different compared with the mRNA level, which was downregulated by 2.7 -fold in the PE placenta compared with the control (Fig. 4C).

\section{Discussion}

$\mathrm{PE}$ is a systemic syndrome of pregnancy characterized by hypertension and proteinuria (22). Although efforts have been made, the etiology of PE is remains to be completely understood. Due to the life-threatening risks and lack of effective treatment, early diagnostic biomarkers of PE are required. Recently, it was reported that the serum concentrations of steroid hormone, including E2, may be associated with PE (23). However, the mechanism of alteration has not been studied. Steroid hormones serve vital roles, including the maintenance of pregnancy in females in addition to regulation of the estrus cycle and puberty (24). Numerous studies have reported that 
A

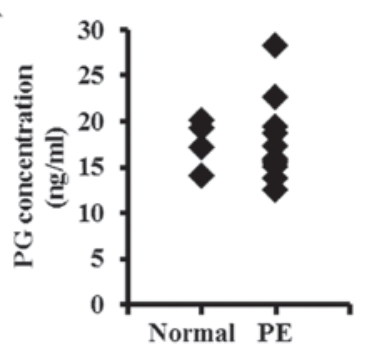

B

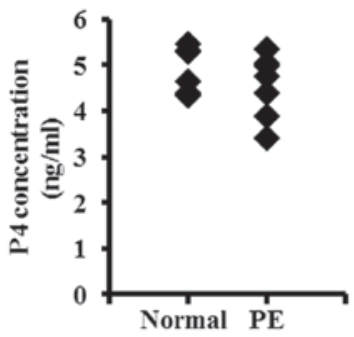

C

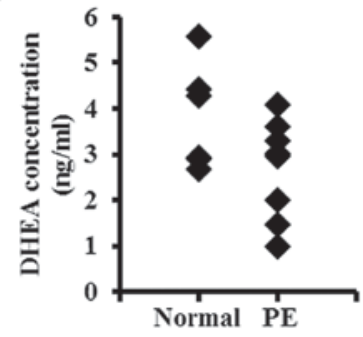

D

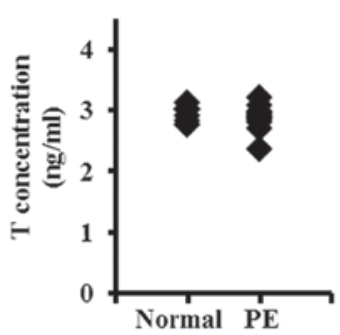

$\mathbf{E}$

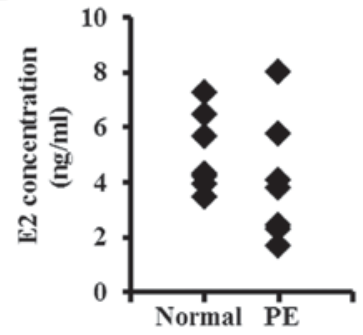

Figure 2. Placental concentration of steroid hormones in women with PE. Steroid hormone concentrations of (A) PG, (B) P4, (C) DHEA, (D) T and (E) E2 were analyzed by ELISA, comparing the normal placenta with the PE placenta. PE, preeclampsia; PG, pregnenolone; P4, progesterone; DHEA, dehydroepiandrosterone; T, testosterone; E2, estrogen.

A

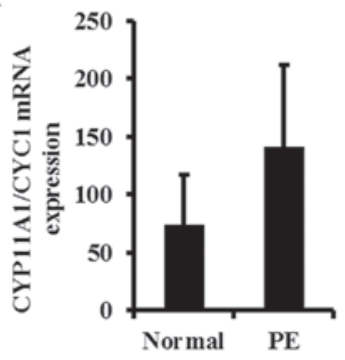

B

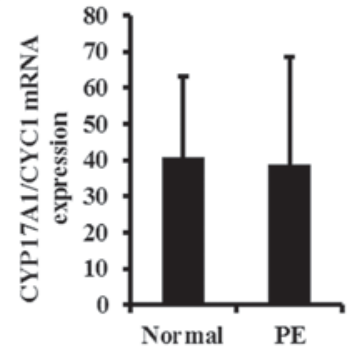

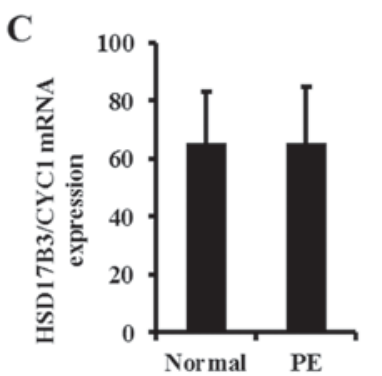
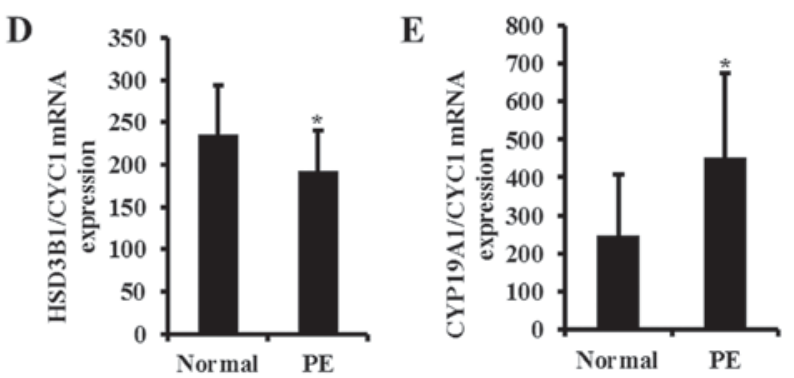

Figure 3. Transcriptional level of steroidogenesis in the human placenta. The mRNA levels of (A) CYP11A1, (B) CYP17A1, (C) HSD17B3, (D) HSD3B1 and (E) CYP19A1, in normal and PE placentas, were analyzed by the reverse transcription-quantitative polymerase chain reaction. Total mRNA was harvested from human normal and PE placentas subsequent to the onset of labor. The total mRNA expression level was normalized to that of CYC1. Data are expressed as the mean \pm standard deviation. "P<0.05 vs. Normal. CYP11A1, cholesterol side-chain cleavage enzyme; CYP17A1, steroid 17- $\alpha$-hydroxylase/17,20 lyase; HSD17B3, testosterone 17- $\beta$-dehydrogenase 3; HSD3B1, $3 \beta$-hydroxysteroid dehydrogenase/ $\delta 54$-isomerase type 1; CYP19A1, aromatase; CYC1, cytochrome c1 heme protein mitochondrial; PE, preeclampsia.

the concentrations of these steroid hormones in PE serum contribute to pathogenesis of PE $(17,18,25,26)$. Since sera are collected from women with different characteristics, including age, body weight and clinical histories, hormone levels vary and the reliability of data is decreased. Therefore, the regulation of steroid hormones in PE women is controversial. For instance, case-control and cross-sectional studies have reported an association between vitamin $\mathrm{D}$ status and $\mathrm{PE}$, although evidence has been inconsistent $(27,28)$. In addition, antenatal corticosteroid therapy in women with PE may be associated with fetal maturation (17). However, the present study focused on sex steroid hormone, since the levels of sex steroid hormones are dynamically regulated during pregnancy. Additionally, there are previous studies demonstrating that sex steroid hormones may 
A
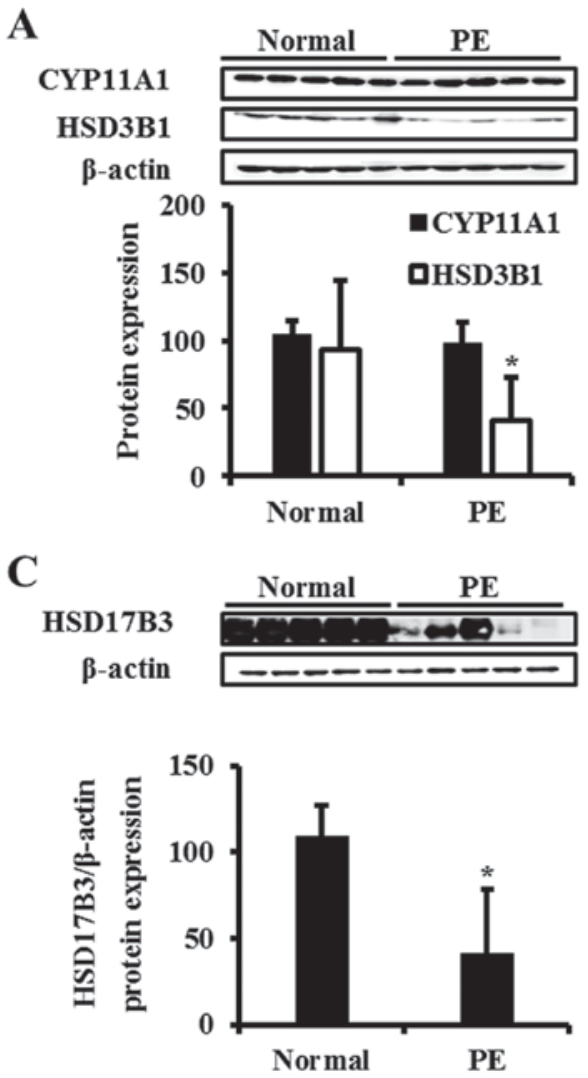

B
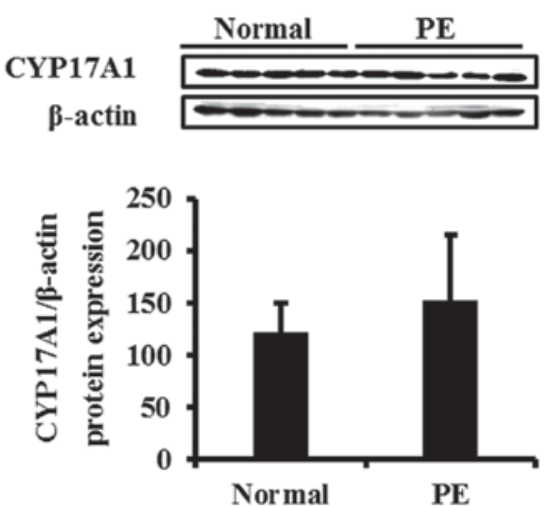

D
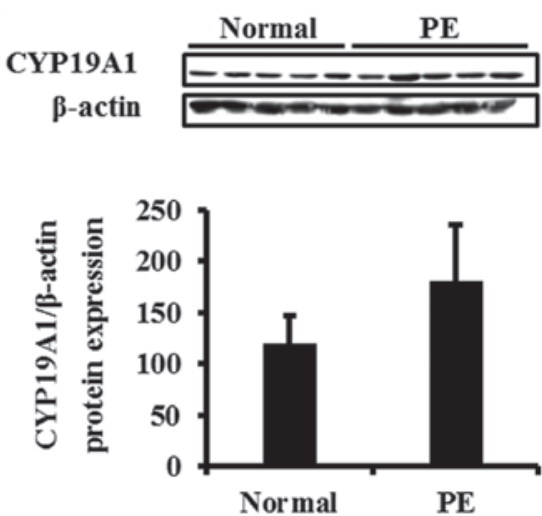

Figure 4. Translational level of steroidogenesis in the human placenta. The protein levels of (A) CYP11A1 and HSD3B1 (B) CYP17A1, (C) HSD17B3 and (D) CYP19A1 in normal and PE placentas were examined. Total proteins were harvested from human normal and PE placentas subsequent to the onset of labor. Proteins were processed for western blot analysis. The total protein expression level was normalized to that of $\beta$-actin. The images of CYP11A1, HSD3B1 and $\beta$-actin were derived from same blot. Data are expressed as the mean \pm standard deviation. "P $<0.05$ vs. Normal. CYP11A1, cholesterol side-chain cleavage enzyme; CYP17A1, steroid 17- $\alpha$-hydroxylase/17,20 lyase; HSD17B3, testosterone 17- $\beta$-dehydrogenase 3; HSD3B1, $3 \beta$-hydroxysteroid dehydrogenase/85 4-isomerase type 1; CYP19A1, aromatase; CYC1, cytochrome $\mathrm{c} 1$ heme protein mitochondrial; $\mathrm{PE}$, preeclampsia.

be associated with PE. A previous study reported that serum levels of DHEA and E2 in patients with PE were decreased compared with those in normal pregnant woman, whereas P4 production was markedly increased in PE (17). However, Iou et al (26) indicated that serum levels of P4 were decreased in women with PE during the third trimester.

In the present study, serum and placental levels of steroid hormones, including PG, P4, DHEA, T and E2, were examined in patients with PE and normal pregnant women. The assessed steroid hormones were selected as they are known to be involved in the regulation of pregnancy. It has been reported that $\mathrm{PG}$ is a precursor of other steroid hormones such as $\mathrm{P} 4$ and DHEA (29) P4 is known to reduce vascular resistance by decreasing the sensitivity of angiotensin and increasing the production of endothelial vasodilator, which directly affects muscles (30). Additionally, P4 stimulates the production and secretion of substances required for the growth and development of the conceptus (31). E2 stimulates the expression of VEGF and angiogenesis in the uterus during normal gestation, serving important roles in implantation, pregnancy maintenance and embryonic development $(11,32)$. DHEA additionally increases vascular endothelial proliferation, migration and vascular tube formation by activating the extracellular signal-regulated kinase 1/2 pathway (33). Therefore, a lack of these steroid hormones may lead to abnormalities in the development of the placenta, leading to the pathogenesis of PE.
According to the results of the present study, the serum levels of P4 and E2 were significantly decreased in women with PE. In addition, PG and DHEA levels were slightly decreased in PE serum samples.

To completely elucidate the mechanism of steroidogenesis, the present study quantified steroid hormones in the human placenta. In normal women, the levels of PG, DHEA and E2 were increased in the serum compared with the placenta, while the levels of $\mathrm{P} 4$ and $\mathrm{T}$ were similar, suggesting that other reproductive organs from the mother or fetus may contribute to the concentrations of hormones. However, the altered patterns of hormones in women with PE in the placenta were similar to the results from the serum. In PE placentas, steroid hormones, apart from $\mathrm{T}$, tended to decrease compared with the normal placenta, and this regulation was even more apparent in the serum. These results indicated that the serum concentration of steroid hormones is primarily regulated by the placenta and that the reduced hormones may contribute the physiological features of women with PE.

For the following experiment, the expression of enzymes associated with synthesis of steroid hormones in the placenta was analyzed. The expression levels of CYP11A1 and CYP17A1 were not altered in PE compared with normal placentas. The serum PG concentration was not altered in women with PE compared with the normal group, and this may be explained by the results of the analysis of CYP11A1 and CYP17A1 gene 
expression. CYP11A1 and CYP17A1 are involved in the process of PG metabolism and their expression was not significantly altered in the PE placenta. Since the expression of HSD3B1, the enzyme converting DHEA to androstenedione, was decreased, it was hypothesized that the concentration of DHEA in the maternal serum may have been enhanced. However, the results of the present study demonstrated that DHEA was decreased in the serum and placenta. During pregnancy, steroidogenesis is a complex process occurring in a number of organs, including the maternal uterus, placenta, fetal membrane, and the maternal and fetal hypothalamic-pituitary-adrenal (HPA) axis, although the placenta is the most important of these. During normal pregnancy, increased production of corticotropin releasing hormone from decidual, trophoblastic and fetal membranes leads to an increase in circulating cortisol in midgestation (34). The fetal HPA axis, activated by cortisol, results in enhanced fetal pituitary adrenocorticotropin hormone secretion, which leads to release of the abundant C19 estrogen precursor DHEA sulfate from the fetal adrenal zone. The results of the present study demonstrated that the serum concentration of DHEA in normal women and patients with PE was increased compared with the placenta, suggesting that the fetal production of DHEA is important for the regulation of maternal DHEA concentration. It has also been reported that the synthesis of DHEA by the fetal adrenal gland is important for placental steroid production (35).

The synthesis of $\mathrm{T}$ from $\mathrm{P} 4$ and DHEA, catalyzed by HSD17B3, was markedly reduced in the PE placenta, suggesting that the products of these enzymes, including E2, P4 and T, may be reduced. Indeed, the hormone data in the present study demonstrated that the concentration of E2 was decreased in the serum and placenta. Notably, the expression of CYP19A1, the final enzyme involved in the synthesis of E2, was enhanced in the PE placenta, in contrast to the reduced concentrations of hormones. This may be a compensatory response of the placental tissue to elevate the serum levels of $\mathrm{E} 2$ in patients with PE.

In conclusion, the results of the present study provided novel insights into the association between steroid hormones and PE. In the PE placenta, the synthesis of steroid hormones, including P4 and E2, was decreased via the regulation of steroidogenic enzymes.

\section{Acknowledgements}

The present study was supported by a grant from the Korea Health Technology R\&D Project through the Korea Health Industry Development Institute (KHIDI), funded by the Ministry of Health and Welfare (grant no. HI16C0313).

\section{References}

1. Ushida T, Kotani T, Tsuda H, Imai K, Nakano T, Hirako S, Ito Y, $\mathrm{Li} \mathrm{H}$, Mano Y, Wang J, et al: Molecular hydrogen ameliorates several characteristics of preeclampsia in the Reduced Uterine Perfusion Pressure (RUPP) rat model. Free Radic Biol Med 101: 524-533, 2016.

2. Venkatesha S, Toporsian M, Lam C, Hanai J, Hanai JI, Mammoto T, Kim YM, Bdolah Y, Lim KH, Yuan HT, et al: Soluble endoglin contributes to the pathogenesis of preeclampsia. Nat Med 12: 642-649, 2006.

3. Duska D and Anja HF: Low catechol-O-methyltransferase and 2-methoxyestradiol in preeclampsia: More than a unifying hypothesis. Nephrol Dial Transplant 24: 31-33, 2009.
4. Tian M, Zhang Y, Liu Z, Sun G, Mor G and Liao A: The PD-1/PD-L1 inhibitory pathway is altered in pre-eclampsia and regulates $\mathrm{T}$ cell responses in pre-eclamptic rats. Sci Rep 6: 27683, 2016.

5. Brownfoot FC, Tong S, Hannan NJ, Binder NK, Walker SP, Cannon P, Hastie R, Onda K and Kaitu'u-Lino TJ: Effects of pravastatin on human placenta, endothelium, and women with severe preeclampsia. Hypertension 66: 687-697, Discussion 445, 2015.

6. Whitley GS and Cartwright JE: Cellular and molecular regulation of spiral artery remodelling: Lessons from the cardiovascular field. Placenta 31: 465-474, 2010.

7. Gunel T, Hosseini MK, Gumusoglu E, Dolekcap I and Aydinli K: Future perspective of preeclampsia by miRNA. Global J Hum Genet Gene Ther 2: 53-67, 2014

8. Zuniga FA, Ormazabal V, Gutierrez N, Aguilera V, Radojkovic C, Veas C, Escudero C, Lamperti L and Aguayo C: Role of lectin-like oxidized low density lipoprotein-1 in fetoplacental vascular dysfunction in preeclampsia. Biomed Res Int 2014: 353616, 2014.

9. Pepe G and Albrecht E: Steroid endocrinology of pregnancy. Glob Libr Women's Med 5: 38, 2008.

10. Young SM, Gryder LK, Zava D, Kimball D and Benyshek DC: Presence and concentration of 17 hormones in human placenta processed for encapsulation and consumption. Placenta 43: 86-89, 2016.

11. Kim SC, Park MN, Lee YJ, Joo JK and An BS: Interaction of steroid receptor coactivators and estrogen receptors in the human placenta. J Mol Endocrinol 56: 239-247, 2016.

12. Miller WL: Steroid hormone synthesis in mitochondria. Mol Cell Endocrinol 379: 62-73, 2013

13. Sato K, Iemitsu M, Matsutani K, Kurihara T, Hamaoka T and Fujita S: Resistance training restores muscle sex steroid hormone steroidogenesis in older men. FASEB J 28: 1891-1897, 2014.

14. Arukwe A: Steroidogenic acute regulatory (StAR) protein and cholesterol side-chain cleavage (P450scc)-regulated steroidogenesis as an organ-specific molecular and cellular target for endocrine disrupting chemicals in fish. Cell Biol Toxicol 24: 527-540, 2008.

15. Miller WL and Auchus RJ: The molecular biology, biochemistry, and physiology of human steroidogenesis and its disorders. Endocr Rev 32: 81-151, 2011.

16. Tremblay JJ: Molecular regulation of steroidogenesis in endocrine Leydig cells. Steroids 103: 3-10, 2015.

17. Hertig A, Liere P, Chabbert-Buffet N, Fort J, Pianose A, Eychennee B, Cambourge A, Schumacher M, Berkane N, Lefevre G, et al: Steroid profiling in preeclamptic women: Evidence for aromatase deficiency. Am J Obstet Gynecol 205: 477.e1-e9, 2010.

18. Zeisler H, Jirecek S, Hohlagschwandtner M, Knöfler M, Tempfer C and Livingston JC: Concentrations of estrogens in patients with preeclampsia. Wien Klin Wochenschr 114: 458-461, 2002.

19. Livak KJ and Schmittgen TD: Analysis of relative gene expression data using real-time quantitative PCR and the 2(-Delta Delta C(T)) method. Methods 25: 402-408, 2001.

20. Clausen T, Slott M, Solvoll K, Drevon CA, Vollset SE and Henriksen T: High intake of energy, sucrose, and polyunsaturated fatty acids is associated with increased risk of preeclampsia. Am J Obstet Gynecol 185: 451-458, 2001.

21. Canakci V, Canakci CF, Canakci H, Canakci E, Cicek Y, Ingec M, Ozgoz M, Demir T, Dilsiz A and Yagiz H: Periodontal disease as a risk factor for pre-eclampsia: A case control study. Aust N Z J Obstet Gynaecol 44: 568-573, 2004.

22. Wang A, Rana S and Karumanchi SA: Preeclampsia: The role of angiogenic factors in its pathogenesis. Physiology (Bethesda) 24: $147-158,2009$.

23. Zheng JJ, Wang HO, Huang M and Zheng FY: Assessment of ADMA, Estradiol, and progesterone in severe preeclmpsia. Clin Exp Hypertens 38: 347-351, 2016.

24. Hong SH, Lee JE, Kim HS, Jung YJ, Hwang D, Lee JH, Yang SY, Kim SC, Cho SK and An BS: Effect of vitamin D3 on production of progesterone in porcine granulosa cells by regulation of steroidogenic enzymes. J Biomed Res 30: 203-208, 2016.

25. Chen D, Dong M, Fang Q, He J, Wang Z and Yang X: Alterations of serum resistin in normal pregnancy and pre-eclampsia. Clin Sci (Lond) 108: 81-84, 2005.

26. Iou SG, Eskandari M and Dabiri A: Evaluation of androgen and progesterone levels of women with preeclampsia in third trimester. Med J Islamic World Acad Sci 15: 19-22, 2005. 
27. Welberg L: Addiciton: Pregnenolone limits effects of cannabis. Nat Rev Neurosci 15: 66-67, 2014.

28. Purswani JM, Gala P, Dwarkanath P, Larkin HM, Kurpad A and Mehta S: The role of vitamin D in pre-eclampsia: A systematic review. BMC Pregnancy Childbirth 17: 231, 2017.

29. Vestgaard M, Secher AL, Ringholm L, Jensen J, Damm P and Mathiesen ER: Vitamin D insufficiency, preterm delivery and preeclampsia in women with type 1 diabetes-an observational study. Acta Obstet Gynecol Scand 96: 1197-1204, 2017.

30. Albrecht ED and Pepe GJ: Estrogen regulation of placental angiogenesis and fetal ovarian development during primate pregnancy. Int J Dev Biol 54: 397-408, 2010.

31. Steinhausera CB, Bazerb FW, Burghardta RC and Johnsona GA: Expression of progesterone receptor in the porcine uterus and placenta throughout gestation: Correlation with expression of uteroferrin and osteopontin. Domest Anim Endocrinol 58: 19-29, 2017.
32. Feng Y, Zhang P, Zhang Z, Shi J, Jiao Z and Shao B: Endocrine disrupting effects of triclosan on the placenta in pregnant rats. PloS One 11: e0154758, 2016.

33. Liu D, Iruthayanathan ML, Homan L, Wang Y, YangL, Wang Y and Dillon JS: Dehydroepiandrosterone stimulates endothelial proliferation and angiogenesis through extracellular signal-regulated kinase 1/2-mediated mechanisms. Endocrinology 149: 889-898, 2008.

34. Lockwood CJ, Radunovic N, Nastic D, Petkovic S, Aigner S and Berkowitz GS: Corticotropin-releasing hormone and related pituitary-adrenal axis hormones in fetal and maternal blood during the second half of pregnancy. J Perinat Med 24: 243-51, 1996.

35. Hoffman Sage Y, Lee L, Thomas AM, Benson CB and Shipp TD: Fetal adrenal gland volume and preterm birth: A prospective third-trimester screening evaluation. J Matern Fetal Neonatal Med 29: 1552-1555, 2016. 